\title{
First syntheses of D-mannose penta- and decasaccharides, the repeating unit and its dimer of the cell-wall mannan of Candida kefyr IFO 0586
}

\author{
Ying Xing and Jun Ning* \\ Research Center for Eco-Environmental Sciences, Chinese Academy of Sciences, PO Box 2871, Beijing 100085, PR China
}

Received 11 January 2003; revised 18 February 2003; accepted 26 February 2003

\begin{abstract}
D-Mannopyranosyl-( $1 \rightarrow 2)-\alpha$-D-mannopyranosyl-( $1 \rightarrow 6)$-[ $\alpha$-D-mannopyranosyl-( $1 \rightarrow 2)-\alpha$-D-mannopyranosyl- $(1 \rightarrow 2)]-\alpha$ D-mannopyranose and $\alpha$-D-mannopyranosyl-( $(1 \rightarrow 2)-\alpha$-D-mannopyranosyl- $(1 \rightarrow 6)-[\alpha$-D-mannopyranosyl- $(1 \rightarrow 2)-\alpha-D-m a n n o p y r a n-$ osyl-( $1 \rightarrow 2)]-\alpha$-D-mannopyranosyl-( $1 \rightarrow 6)-[\alpha-D-m a n n o p y r a n o s y l-(1 \rightarrow 2)]-\alpha$-D-mannopyranosyl-(1

$\rightarrow 6$ )-[ $\alpha$-D-mannopyranosyl-( $1 \rightarrow 2)-\alpha$-D-mannopyranosyl-( $(1 \rightarrow 2)]-\alpha$-D-mannopyranose, the repeating unit and its dimer of the cell wall mannan of the pathogenic yeast Candida kefyr IFO 0586, have been efficiently synthesized via their allyl glycosides by using allyl 3,4,6-tri- $O$-benzoyl- $\alpha$-D-mannopyranoside, allyl 6- $O$-acetyl-3,4-di- $O$-benzoyl- $\alpha$-D-mannopyranoside, and allyl 3,4-di- $O$-benzoyl- $\alpha$-D-mannopyranoside as synthons. The blocked pentasaccharide was regio- and stereoselectively prepared by coupling of allyl 3,4-di- $O$-benzoyl- $\alpha$-D-mannopyranoside with 2,3,4,6-tetra- $O$-benzoyl- $\alpha$-D-mannopyranosyl-( $1 \rightarrow 2$ )-6- $O$-acetyl-3,4-di- $O$-benzoyl- $\alpha$-D-mannopyranosyl trichloroacetimidate, and then with 2,3,4,6-tetra- $O$-benzoyl- $\alpha$-D-mannopyranosyl-(1 $\rightarrow 2)-3,4,6$-tri- $O$ benzoyl- $\alpha$-D-mannopyranosyl trichloroacetimidate in a one-pot manner. (C) 2003 Elsevier Science Ltd. All rights reserved.
\end{abstract}

\section{Introduction}

Candida species are opportunistic pathogens of humans that frequently cause severe systemic infections in patients with AIDS, ${ }^{1}$ cancer, ${ }^{2}$ and burns ${ }^{3}$ as well as in those under immunosuppressive or radiation therapy. ${ }^{4}$ An impressive feature of these fungi is that they synthesize cell wall polysaccharides containing predominantly mannose residues. ${ }^{5-7}$ It has been reported that the mannose oligosaccharides present in the fungal D-mannans deeply influence many fundamental biological processes in the organisms. The $\alpha$-linked oligo-D-mannosyl side chains of cell-wall mannan of the pathogenic yeast Candida species play important roles in the binding of yeast cells to the marginal zone of mouse spleen ${ }^{8}$ and in the processes of several types of yeast flocculation. ${ }^{9}$ The alkali-released $\alpha$-linked D-manno-oligosaccharides obtained from a Candida albicans cell-wall mannan were potent inhibitors of lymphoproliferation induced by the parent D-mannan. ${ }^{10,11}$ Furthermore, many of them (if not all) are antigenically relevant. Hence, there is a great need for usable quantities of the

\footnotetext{
* Corresponding author. Tel.: 8610-62849157; fax: 8610-62923563; e-mail: jning@mail.rcees.ac.cn
}

natural oligosaccharides with a well-defined structure and composition for biological studies aiming at a better understanding of these phenomena at the molecular level. Given the intrinsic difficulty in obtaining complex natural oligosaccharides and glycoconjugates in a pure and homogeneous form from natural sources because of the presence of mixtures of glycosylated species (glycoforms). A major opportunity is provided by chemical synthesis.

As part of our continuing efforts dedicated to developing efficient strategies for the construction of sugars, we have prepared a lot of oligosaccharides with various structures present in natural sources including fungi. ${ }^{12-16}$ In 1994, Kobayashi et al. ${ }^{17}$ conducted a structural analysis of an antigenic cell wall mannan isolated from the pathogenic yeast Candida kefyr IFO 0586 and found that this sugar has a long $\alpha-(1 \rightarrow 6)$-linked $D$ mannopyranosyl backbone and many short $\alpha-(1 \rightarrow 2)$ linked D-mannopyranosyl side-chains in a comb-like structure as shown in Figure 1. The reasons mentioned above, together with the fact that there have been no reports dealing with the synthesis of these highly branched oligosaccharides, prompted us to develop an efficient method for the synthesis of this kind of complex carbohydrate. The preparation of allyl- $\alpha$-D-penta- 

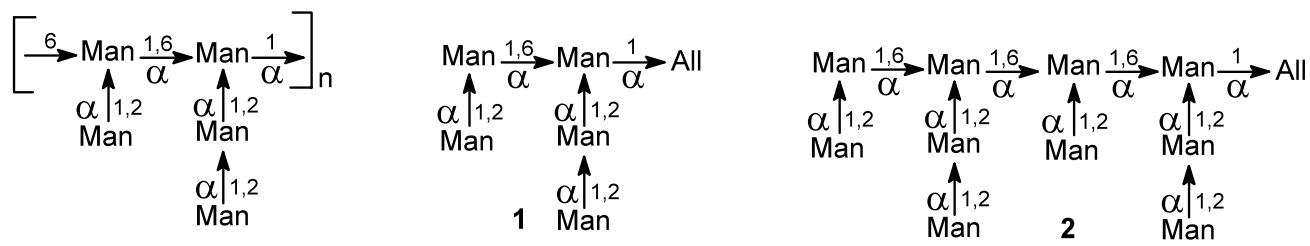

Figure 1. The structures of the cell-wall mannan of Candida kefyr IFO 0586 and the synthesized oligosaccharides 1 and 2.

mannoside 1 and allyl- $\alpha$-D-decamannoside 2 (Fig. 1), the repeating unit and its dimer of the cell wall mannan of the pathogenic yeast Candida kefyr IFO 0586 are presented as typical examples using the strategy developed here.

\section{Results and discussion}

For the synthesis of the complex decasaccharide 2, we needed 2,3,4,6-tetra- $O$-benzoyl-D-mannopyranosyl trichloroacetimidate 3, allyl 3,4,6-tri- $O$-benzoyl-D-mannopyranoside 5, allyl 6- $O$-acetyl-3,4-di- $O$-benzoyl- $\alpha$-Dmannopyranoside 7, and allyl 3,4-di- $O$-benzoyl-Dmannopyranoside $\mathbf{9}$ as building materials. Compound $\mathbf{3}$ was prepared according to the reported procedure. ${ }^{18}$ The glycosyl acceptor $\mathbf{5}$ was obtained from coupling of 2- $O$-acetyl-3,4,6-tri- $O$-benzoyl-D-mannopyranosyl trichloroacetimidate $\mathbf{4}^{18}$ with allyl alcohol followed by $2-O$-deacetylation in $87 \%$ yield (Scheme 1). The glycosyl acceptors 7 and 9 were derived from 2,6-di- $O$-acetyl3,4-di- $O$-benzoyl-D-mannopyranosyl trichloroacetimidate 6 which was made according to the method developed in our laboratory. ${ }^{16}$ We were lucky to find that coupling of 6 with allyl alcohol in the presence of TMSOTf ( 0.3 equiv.) gave two compounds; one was the required glycosyl acceptor 7 in $31 \%$, and another was allyl 2,6-di- $O$-acetyl-3,4-di- $O$-benzoyl-D-mannopyranoside 8 in $63 \%$ yield. During the glycosylation process of 6 with allyl alcohol, some of the 2-O-acetyl groups of 6 were removed, and it was observed that with the increase of the amount of TMSOTf added to the reaction system, the 2-O-acetyl group removed product 7 was increased. Selective removal of the acetyl groups of 8 in $\mathrm{MeOH}$ containing $0.5 \% \mathrm{HCl}$ gave the glycosyl acceptor 9 in $93 \%$ yield.
Condensation of $\mathbf{3}$ and $\mathbf{5}$ with TMSOTf as catalyst and $4 \AA$ molecular sieves in $\mathrm{CH}_{2} \mathrm{Cl}_{2}$ at $\mathrm{rt}$ afforded the disaccharides 10 in $88 \%$ yield. Deallylation of 10 with $\mathrm{PdCl}_{2}$ followed by activation with $\mathrm{CCl}_{3} \mathrm{CN}$ in the presence of DBU, afforded the disaccharide donor 11 in $80 \%$ yield (for two steps) (Scheme 2). Similar procedures gave another disaccharide donor $\mathbf{1 3}$.

Coupling of 9 and 13 with TMSOTf as catalyst and $4 \AA$ molecular sieves in $\mathrm{CH}_{2} \mathrm{Cl}_{2}$ at $-45^{\circ} \mathrm{C}$ regio- and stereoselectively afforded the trisaccharides $\mathbf{1 4}$ in $91 \%$ yield (Scheme 3). No $(1 \rightarrow 2)$-linked trisaccharide was detected both from ${ }^{1} \mathrm{H}$ NMR and TLC. Acetylation of 14 confirmed 6- $O$-glycosylation as the ${ }^{1} \mathrm{H}$ NMR spectrum of acetylated trisaccharide $\mathbf{1 5}$ showed a newly emerged doublet of doublets at $\delta 5.54 \mathrm{ppm}$ for $\mathrm{H}-2$. Condensation of 14 with 11 afforded the blocked pentasaccharide $\mathbf{1 6}$ in $90 \%$ yield. The ${ }^{1} \mathrm{H}$ NMR data of $\mathbf{1 6}$ contained structurally characteristic information, i.e. one acetyl signal ( $\delta$ 1.95), one allyl signal $(\delta$ 5.43-5.27) and five $\mathrm{H}-1$ signals $(\delta 5.60,5.58,5.39,5.27$, and 5.12). The ${ }^{13} \mathrm{C}$ NMR spectrum of $\mathbf{1 6}$ gave five signals for $\mathrm{C}-1$ $(100.04,99.65,99.42,98.36,98.04)$ with ${ }^{2} J_{\mathrm{C} 1-\mathrm{H} 1}$ from 170 to $176.8 \mathrm{~Hz}$ indicating complete $\alpha$-linkages. Encouraged by the smooth coupling reaction results, a one-pot procedure for the synthesis of the pentasaccharide 16 was carried out. Thus, treatment of 9 and $\mathbf{1 3}$ with TMSOTf as catalyst and $4 \AA$ molecular sieves in $\mathrm{CH}_{2} \mathrm{Cl}_{2}$ at $-45^{\circ} \mathrm{C}$, followed by addition of $\mathbf{1 1}$ at $\mathrm{rt}$ afforded compound $\mathbf{1 6}$ in $83 \%$ yield. Selective removal of the 6-O-acetyl group of the pentasaccharide $\mathbf{1 6}$ gave the glycosyl accepter 18. Deallylation of 16 with $\mathrm{PdCl}_{2}$ followed by activation with $\mathrm{CCl}_{3} \mathrm{CN}$ in the presence of DBU gave the pentasaccharide donor 17. The fully protected decasaccharide 19 was obtained by coupling $\mathbf{1 7}$ with $\mathbf{1 8}$ in $50 \%$ yield. The ${ }^{1} \mathrm{H}$ NMR data of $\mathbf{1 9}$
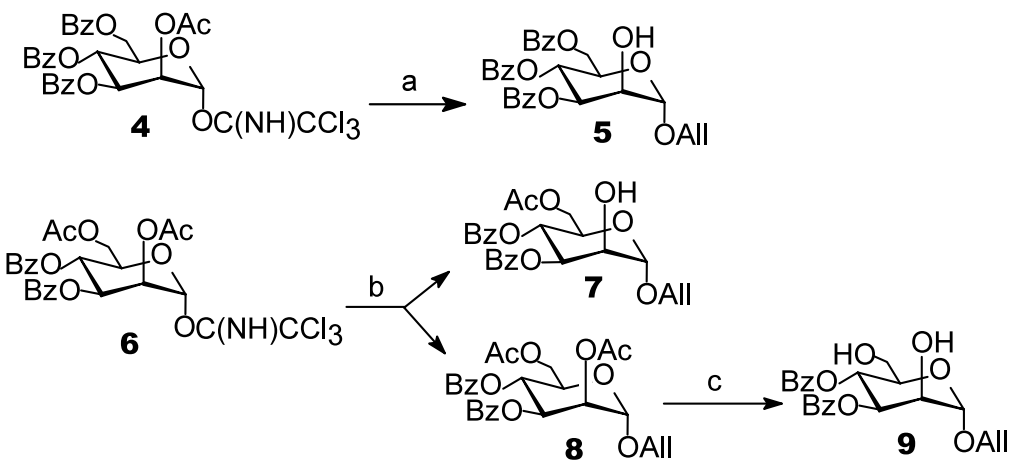

Scheme 1. Reagents and conditions: (a) i. allyl alcohol (2 equiv.), TMSOTf ( 0.1 equiv.), $\mathrm{CH}_{2} \mathrm{Cl}_{2}, \mathrm{rt}, 1 \mathrm{~h}$; ii. methanol/0.5\% $\mathrm{HCl}$, rt, $12 \mathrm{~h}, 87 \%$ (over the two steps); (b) allyl alcohol (2 equiv.), TMSOTf (0.3 equiv), $\mathrm{CH}_{2} \mathrm{Cl}_{2}, \mathrm{rt}, 1 \mathrm{~h}, 31 \%$ for 7 and $63 \%$ for $\mathbf{8}$; (c) methanol $/ 0.5 \% \mathrm{HCl}, \mathrm{rt}, 12 \mathrm{~h}, 93 \%$. 

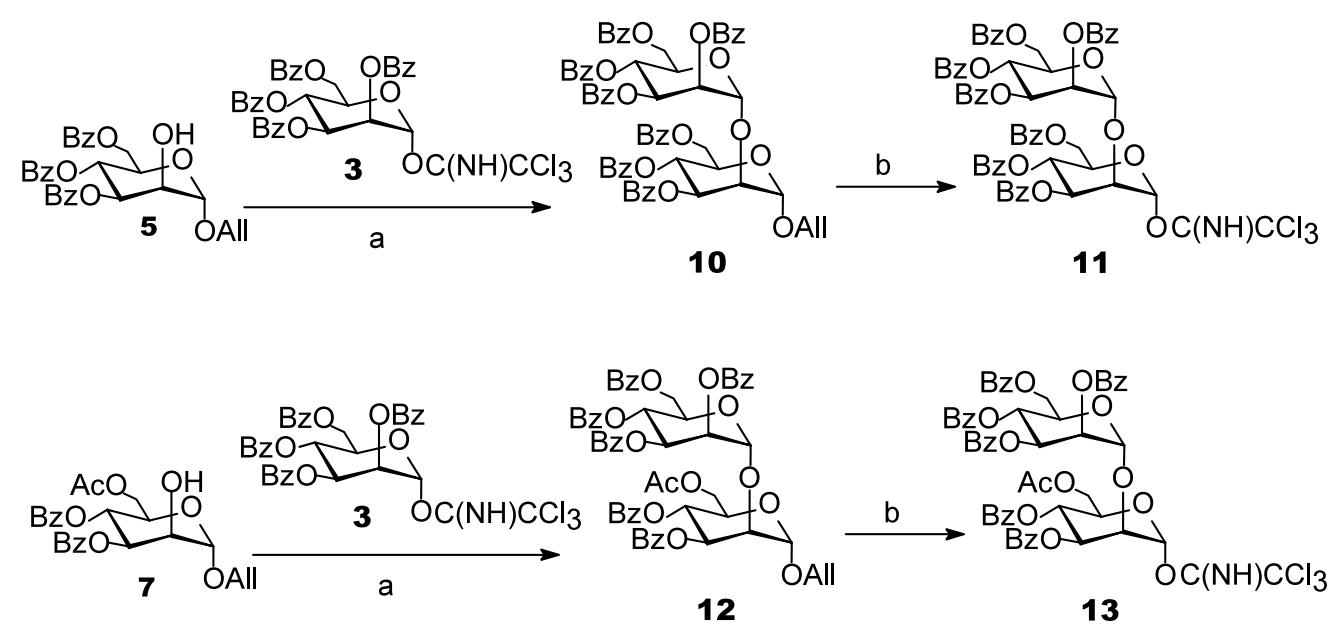

Scheme 2. Reagents and conditions: (a) $\mathrm{CH}_{2} \mathrm{Cl}_{2}$, TMSOTf (cat.), rt, $1 \mathrm{~h}, 88 \%$ for $\mathbf{1 0}$ and $92 \%$ for 12; (b) i. $\mathrm{PdCl}_{2}, \mathrm{CH}_{3} \mathrm{OH}, \mathrm{rt}, 4$ h; ii. $\mathrm{CCl}_{3} \mathrm{CN}, \mathrm{DBU}, \mathrm{CH}_{2} \mathrm{Cl}_{2}, \mathrm{rt}, 2 \mathrm{~h}, 80 \%$ for $\mathbf{1 1}$ and $81 \%$ for $\mathbf{1 3}$ (over the two steps).

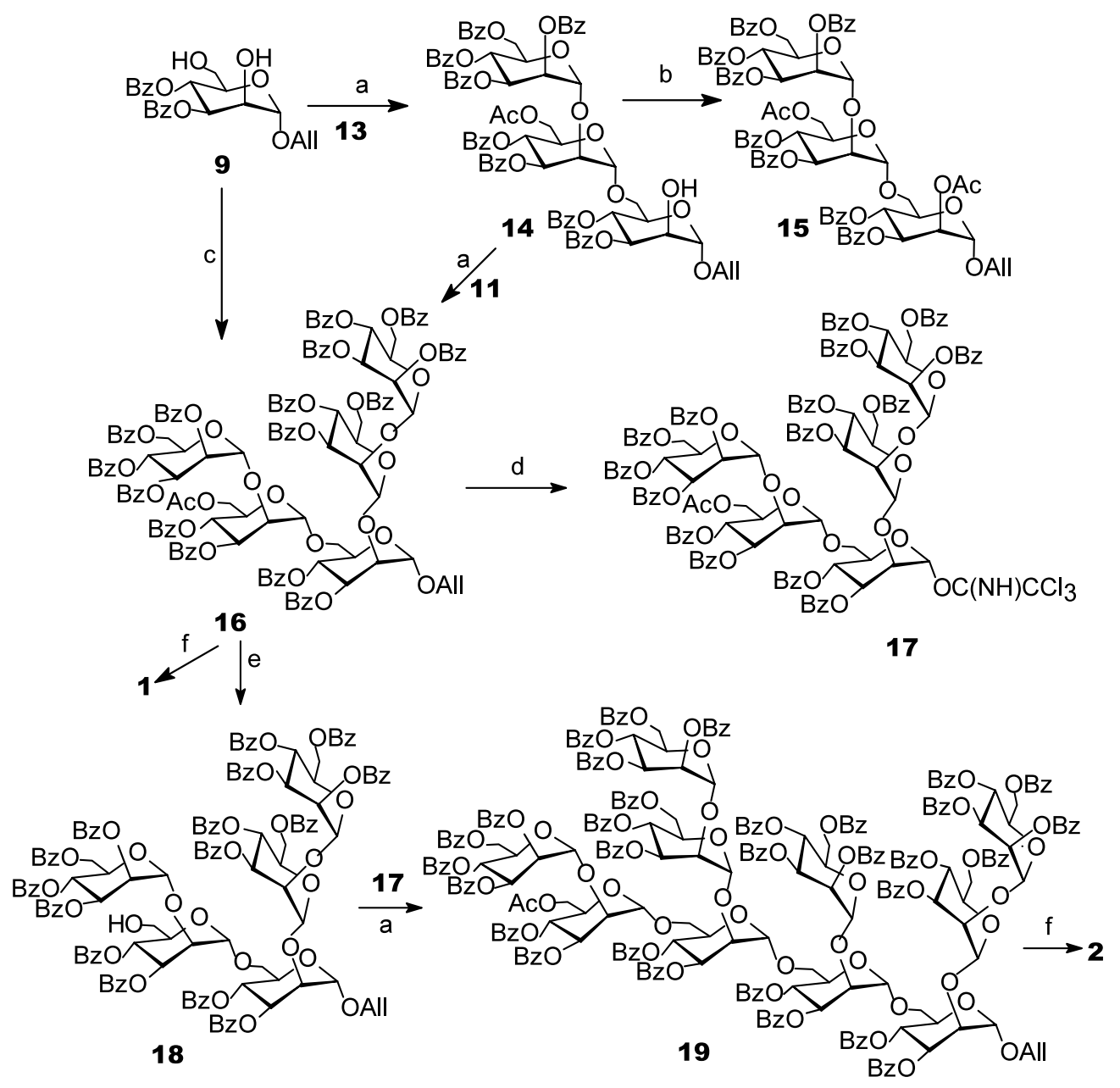

Scheme 3. Reagents and conditions: (a) $\mathrm{CH}_{2} \mathrm{Cl}_{2}$, TMSOTf (cat.), rt, $1 \mathrm{~h}, 91 \%$ for 14, 90\% for 16, 50\% for 19; (b) Ac $\mathrm{O}_{2} \mathrm{O}$, pyridine, rt, 3 h, 100\%; (c) i. 13 (1.0 equiv.), TMSOTf (cat.), $\mathrm{CH}_{2} \mathrm{Cl}_{2}$, MS $4 \AA$ A Powder, $-45^{\circ} \mathrm{C}-\mathrm{rt}, 1.5$ h; ii. 11 (1.2 equiv.), rt, 1 h, $83 \%$ (over the two steps); (d) i. $\mathrm{PdCl}_{2}, \mathrm{CH}_{3} \mathrm{OH}-\mathrm{CH}_{2} \mathrm{Cl}_{2}, 4 \mathrm{~h}$; ii: $\mathrm{CCl}_{3} \mathrm{CN}, \mathrm{DBU}, \mathrm{CH}_{2} \mathrm{Cl}_{2} 2 \mathrm{~h}, 85 \%$ (over the two steps); (e) methanol/0.5\% $\mathrm{HCl}, \mathrm{rt}, 12 \mathrm{~h}, 92 \%$; (f) $\mathrm{CH}_{3} \mathrm{OH}-\mathrm{CH}_{2} \mathrm{Cl}_{2}$ (9:1) satd with dry $\mathrm{NH}_{3}, 40^{\circ} \mathrm{C}, 24 \mathrm{~h}, 91 \%$ for 1, 95\% for 2.

contained structurally characteristic information, i.e. one acetyl signal ( $\delta$ 1.87), one allyl signal ( $\delta$ 5.36-5.20) and ten H-1 signals $(\delta$ 5.70, 5.62, 5.62, 5.48, 5.39, 5.32,
5.28, 5.24, 5.20, and 4.98). The ${ }^{13} \mathrm{C}$ NMR spectrum of 19 gave ten signals for C-1 $(100.07,99.99,99.72,99.55$, 99.43, 99.34, 98.89, 98.26, 98.19, 98.17). Deprotection 
of 16 and 19 using $\mathrm{NH}_{3}$ in $\mathrm{CH}_{3} \mathrm{OH}-\mathrm{CH}_{2} \mathrm{Cl}_{2}$ gave compounds 1 and 2. The NMR spectra of the $\mathbf{1}$ and $\mathbf{2}$ are shown in Figures 2-5.

\section{Conclusion}

In summary, a highly efficient and concise synthesis of the mannose pentasaccharide and decasaccharide of the cell-wall mannan of the pathogenic yeast Candida kefyr IFO 0586 was achieved by regio- and stereoselective glycosylation using glycosyl trichloroimidates as the donors and partially protected sugars as the acceptors. The sole use of acyl groups in the synthesis substantially simplified the procedure. This method should be useful for the synthesis of other complex mannose oligosaccharides in the future.

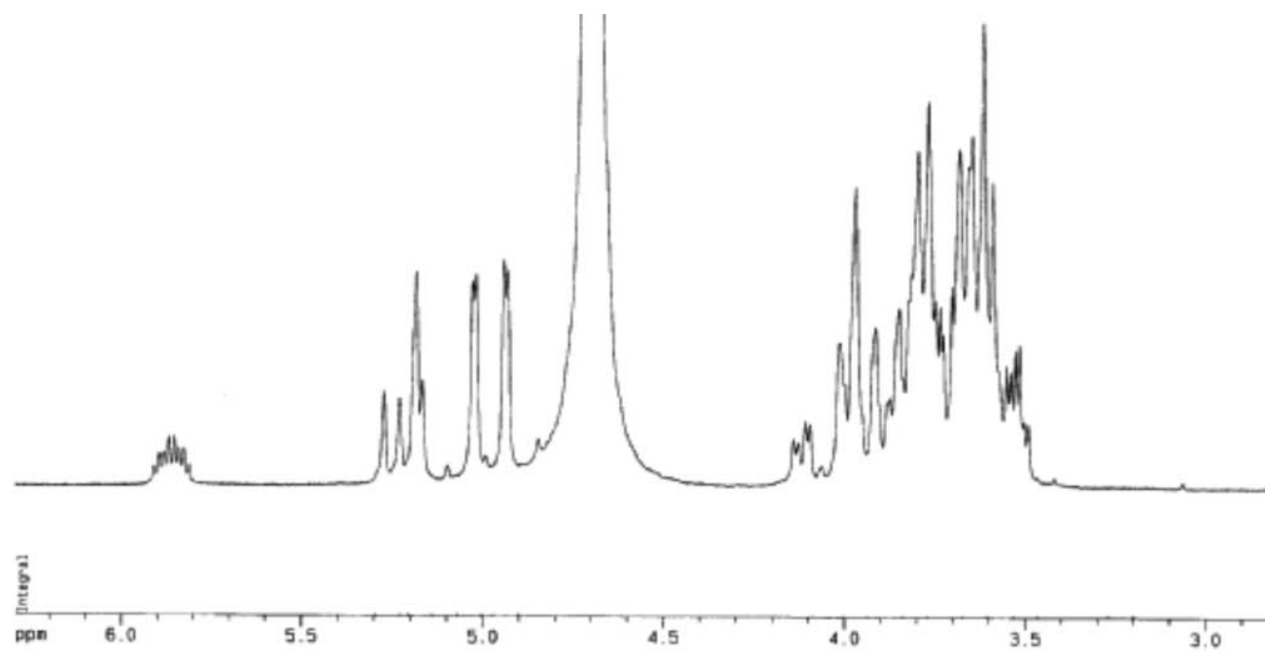

Figure 2. ${ }^{1} \mathrm{H}$ NMR spectrum of compound 1 (solvent $\mathrm{D}_{2} \mathrm{O}$ at $25^{\circ} \mathrm{C}, 400 \mathrm{MHz}$ Bruker ARX 400 spectrometer).

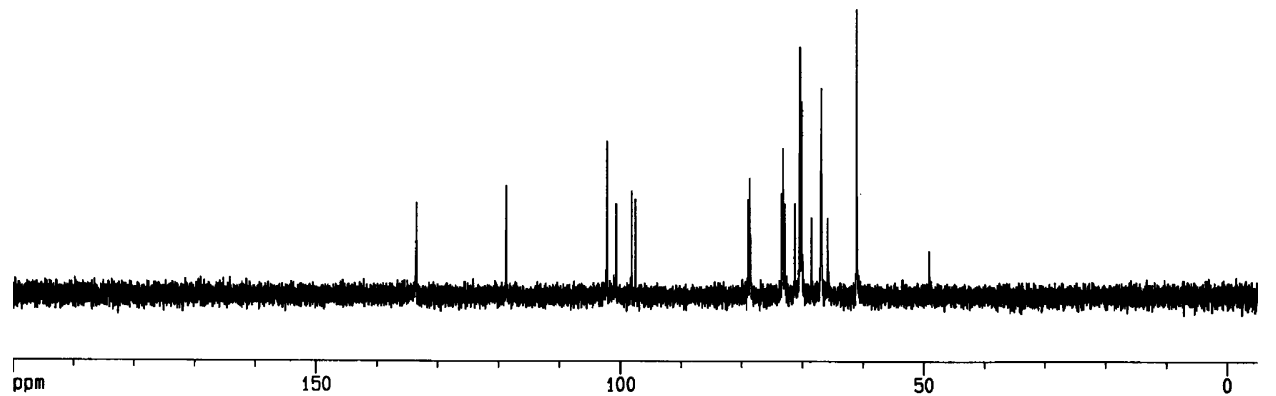

Figure 3. ${ }^{13} \mathrm{C}$ NMR spectrum of compound 1 (solvent $\mathrm{D}_{2} \mathrm{O}$ at $25^{\circ} \mathrm{C}, 100 \mathrm{MHz}$ Bruker ARX 400 spectrometer).

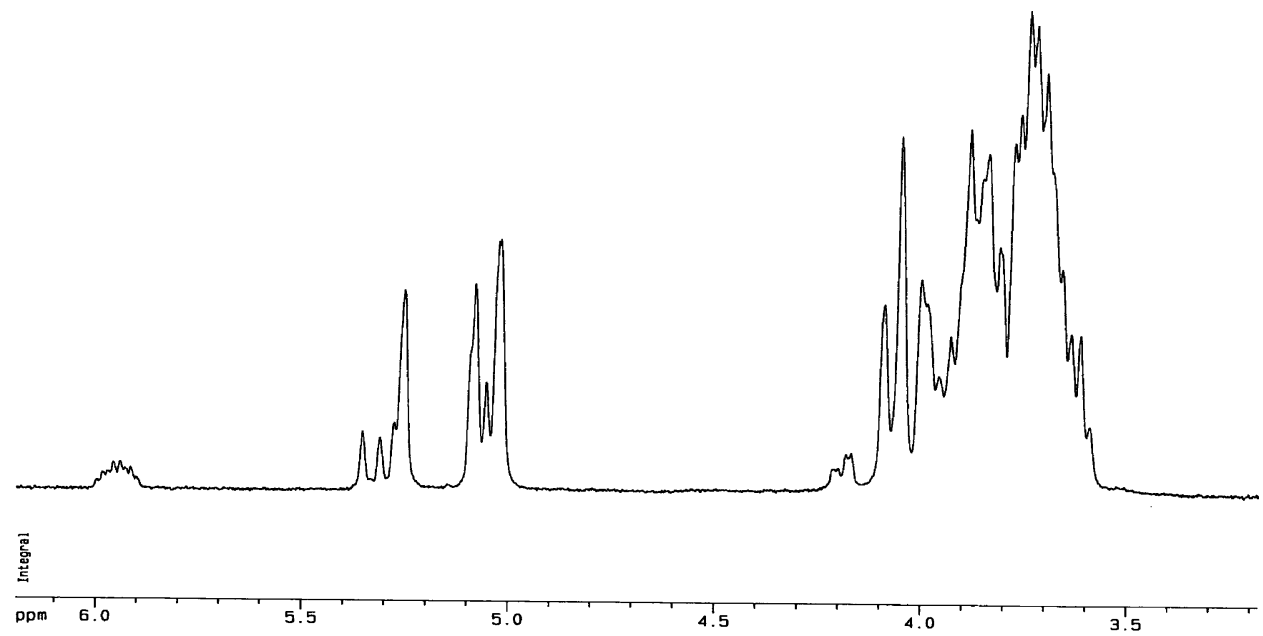

Figure 4. ${ }^{1} \mathrm{H}$ NMR spectrum of compound 2 (solvent $\mathrm{D}_{2} \mathrm{O}$ at $25^{\circ} \mathrm{C}, 400 \mathrm{MHz}$ Bruker ARX 400 spectrometer). 


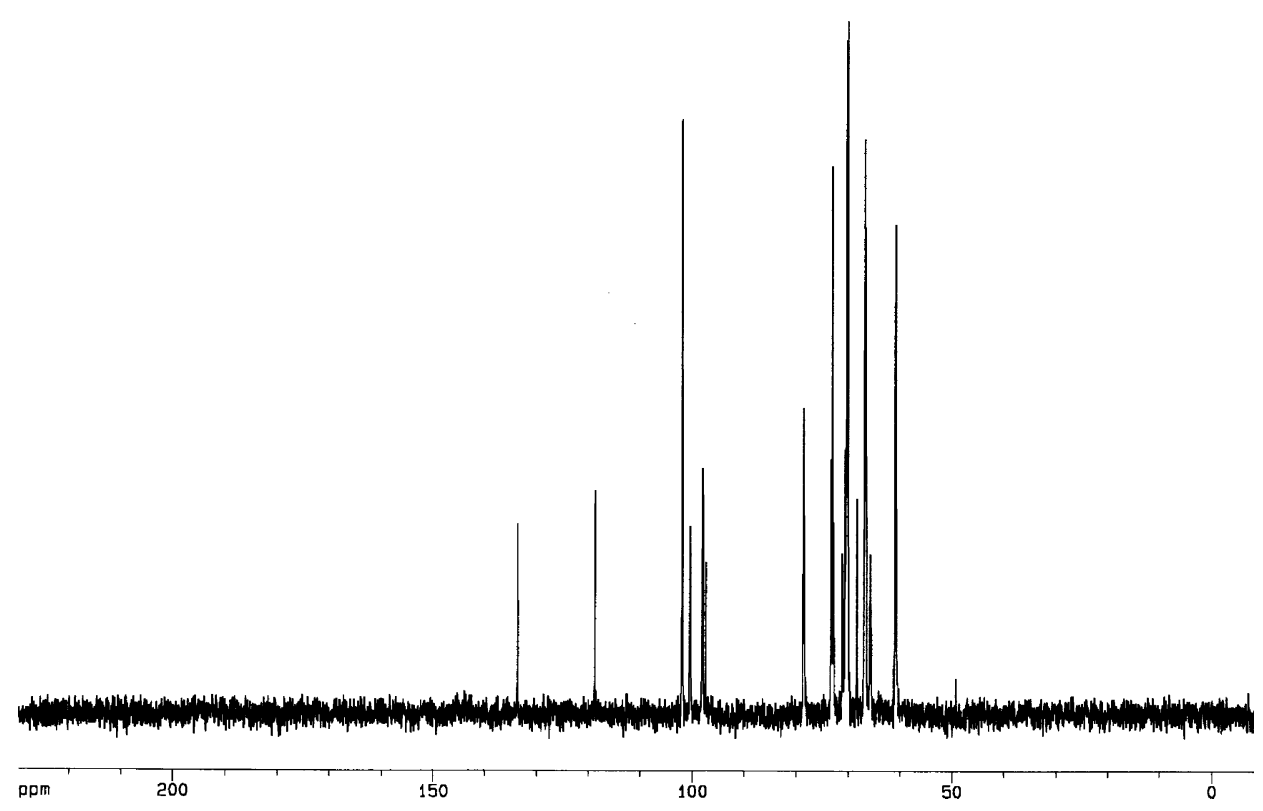

Figure 5. ${ }^{13} \mathrm{C}$ NMR spectrum of compound 2 (solvent $\mathrm{D}_{2} \mathrm{O}$ at $25^{\circ} \mathrm{C}, 100 \mathrm{MHz}$ Bruker $\mathrm{ARX} 400$ spectrometer).

\section{Experimental}

\subsection{General methods}

Optical rotations were determined at $25^{\circ} \mathrm{C}$ with a Perkin-Elmer Model 241-Mc automatic polarimeter. Melting points were determined with a 'Mel-Temp' apparatus. ${ }^{1} \mathrm{H}$ NMR and ${ }^{13} \mathrm{C}$ NMR spectra were recorded with Bruker ARX 400 spectrometers (400 $\mathrm{MHz}$ for ${ }^{1} \mathrm{H}, 100 \mathrm{MHz}$ for ${ }^{13} \mathrm{C}$ ) for solutions in $\mathrm{CDCl}_{3}$ or $\mathrm{D}_{2} \mathrm{O}$ as indicated. Chemical shifts are given in $\mathrm{ppm}$ downfield from internal $\mathrm{Me}_{4} \mathrm{Si}$. Mass spectra were measured using MALTI-TOF-MS with CCA as matrix. Thin-layer chromatography (TLC) was performed on silica gel $\mathrm{HF}_{254}$ with detection by charring with $30 \%$ (v/v) $\mathrm{H}_{2} \mathrm{SO}_{4}$ in $\mathrm{MeOH}$ or in some cases by a UV detector. Column chromatography was conducted by elution of a column $(16 \times 240 \mathrm{~mm}, 18 \times 300 \mathrm{~mm}, 35 \times 400$ $\mathrm{mm})$ of silica gel (100-200 mesh) with EtOAcpetroleum ether $\left(60-90^{\circ} \mathrm{C}\right)$ as the eluent. Solutions were concentrated at $<60^{\circ} \mathrm{C}$ under reduced pressure.

\subsection{Allyl 3,4,6-tri- $O$-benzoyl- $\alpha$-D-mannopyranoside 5}

A solution of 2- $O$-acetyl-3,4,6-tri- $O$-benzoyl- $\alpha$-Dmannopyranosyl trichloroacetimidate 4 (4.3 g, 6.4 mmol) and allyl alcohol $(0.7 \mathrm{ml}, 10 \mathrm{mmol})$ in dry $\mathrm{CH}_{2} \mathrm{Cl}_{2}(50 \mathrm{~mL})$ was stirred with dried molecular sieves (4 $\mathrm{A}, 1 \mathrm{~g})$ under $\mathrm{N}_{2}$ for $15 \mathrm{~min}$, and then TMSOTf (135 $\mu \mathrm{L}, 0.7 \mathrm{mmol}$ ) was added. After $1 \mathrm{~h}$, the reaction mixture was neutralized with $\mathrm{Et}_{3} \mathrm{~N}$, filtered and the filtrate was concentrated. The resulting residue without purification was directly dissolved in $\mathrm{MeOH}(150 \mathrm{~mL})$ containing $0.5 \% \mathrm{HCl}$. The solution was stirred at $\mathrm{rt}$ for $12 \mathrm{~h}$, at the end of which time TLC $(2: 1$ petroleum ether-EtOAc) indicated that the starting material had disappeared. The mixture was neutralized with $\mathrm{Et}_{3} \mathrm{~N}$ and then concentrated to dryness. The residue was partitioned between water and $\mathrm{CH}_{2} \mathrm{Cl}_{2}$, the organic layer was dried over $\mathrm{Na}_{2} \mathrm{SO}_{4}$ and concentrated to a syrup. Purification of the residue by flash chromatography (2:1 petroleum ether-EtOAc) gave 5 as a syrup (2.9 $\mathrm{g}, 87 \%$ for the two steps): $[\alpha]_{\mathrm{D}}-15.1\left(\mathrm{c} 1.0, \mathrm{CHCl}_{3}\right) ;{ }^{1} \mathrm{H}$ NMR $\left(\mathrm{CDCl}_{3}, 400 \mathrm{MHz}\right): \delta 8.03-7.33(\mathrm{~m}, 15 \mathrm{H}, 3$ $\mathrm{Ph} H), 5.95\left(\mathrm{~m}, 1 \mathrm{H}, \mathrm{CH}=\mathrm{CH}_{2}\right), 5.94\left(\mathrm{dd}, 1 \mathrm{H}, J_{3,4}=\right.$ $\left.J_{4,5}=9.8 \mathrm{~Hz}, \mathrm{H}-4\right), 5.70\left(\mathrm{dd}, 1 \mathrm{H}, J_{2,3}=3.1 \mathrm{~Hz}, J_{4,5}=9.8\right.$ $\mathrm{Hz}, \mathrm{H}-3), 5.33$ (dd, $1 \mathrm{H},{ }^{2} J=1.5 \mathrm{~Hz},{ }^{3} J_{\text {trans }}=17.2 \mathrm{~Hz}$, $\left.\mathrm{CH}=\mathrm{CH}_{2}\right), 5.24\left(\mathrm{dd}, 1 \mathrm{H},{ }^{2} \mathrm{~J}=1.5 \mathrm{~Hz},{ }^{3} J_{\text {cis }}=10.4 \mathrm{~Hz}\right.$, $\left.\mathrm{CH}=\mathrm{CH}_{2}\right), 5.03\left(\mathrm{~d}, 1 \mathrm{H}, J_{1,2}=1.6 \mathrm{~Hz}, \mathrm{H}-1\right), 4.58(\mathrm{dd}$, $1 \mathrm{H}, \mathrm{H}-6), 4.48$ (dd, $\left.1 \mathrm{H}, \mathrm{H}^{\prime} 6^{\prime}\right), 4.40-4.08$ (m, $4 \mathrm{H}$, $\left.\mathrm{CH}_{2} \mathrm{CH}=\mathrm{CH}_{2}, \mathrm{H}-2,5\right)$. Anal. calcd for $\mathrm{C}_{30} \mathrm{H}_{28} \mathrm{O}_{9}: \mathrm{C}$, 67.66; H, 5.30. Found: C, 67.93; H, 5.27.

4.3. Allyl 6-O-acetyl-3,4-di- $O$-benzoyl- $\alpha$-D-mannopyranoside 7 and Allyl 2,6-di- $O$-acetyl-3,4-di- $O$-benzoyl- $\alpha$-Dmannopyranoside 8

A solution of 2,6-di- $O$-acetyl-3,4-di- $O$-benzoyl- $\alpha$-Dmannopyranosyl trichloroacetimidate $\mathbf{6}$ (11 g, 17.8 mmol) and allyl alcohol $(2.4 \mathrm{~mL}, 35 \mathrm{mmol})$ in $\mathrm{CH}_{2} \mathrm{Cl}_{2}$ (200 mL) was stirred with dried molecular sieves $(4 \AA$, $4 \mathrm{~g})$ under $\mathrm{N}_{2}$ for $15 \mathrm{~min}$, and then TMSOTf $(1.0 \mathrm{~mL}$, $5.3 \mathrm{mmol}$ ) was added. After $1 \mathrm{~h}$, the reaction mixture was neutralized with $\mathrm{Et}_{3} \mathrm{~N}$, filtered and the filtrate was concentrated. Purification of the residue by chromatography (3:1 petroleum ether-EtOAc) gave allyl 6- $O$-acetyl-3,4-di- $O$-benzoyl- $\alpha$-D-mannopyranoside $7(2.6 \mathrm{~g}$, $31 \%$ ) and allyl 2,6-di- $O$-acetyl-3,4-di- $O$-benzoyl- $\alpha-\mathrm{D}-$ mannopyranoside $8(5.7 \mathrm{~g}, 63 \%)$. For $7:[\alpha]_{\mathrm{D}}-13.4(c$ 1.0, $\left.\mathrm{CHCl}_{3}\right) ;{ }^{1} \mathrm{H}$ NMR (400 $\left.\mathrm{MHz}, \mathrm{CDCl}_{3}\right): \delta$ 7.97-7.26 $(\mathrm{m}, 10 \mathrm{H}, 2 \mathrm{BzH}), 5.98\left(\mathrm{~m}, 1 \mathrm{H}, \mathrm{CH}=\mathrm{CH}_{2}\right), 5.85(\mathrm{dd}$, $\left.1 \mathrm{H}, J_{3,4}=J_{4,5}=10 \mathrm{~Hz}, \mathrm{H}-4\right), 5.65\left(\mathrm{dd}, 1 \mathrm{H}, J_{2,3}=3.0 \mathrm{~Hz}\right.$, $\left.J_{3,4}=10 \mathrm{~Hz}, \mathrm{H}-3\right), 5.37\left(\mathrm{dd}, 1 \mathrm{H},{ }^{2} J=1.5 \mathrm{~Hz},{ }^{3} J_{\text {trans }}=\right.$ $\left.17.2 \mathrm{~Hz}, \mathrm{CH}=\mathrm{CH}_{2}\right), 5.27\left(\mathrm{dd}, 1 \mathrm{H},{ }^{2} \mathrm{~J}=1.5 \mathrm{~Hz},{ }^{3} J_{\text {cis }}=\right.$ $\left.10.4 \mathrm{~Hz}, \mathrm{CH}=\mathrm{CH}_{2}\right), 5.00\left(\mathrm{~d}, 1 \mathrm{H}, J_{1,2}=1.5 \mathrm{~Hz}, \mathrm{H}-1\right)$, 4.34-4.19 (m, $\left.5 \mathrm{H}, \mathrm{CH}_{2} \mathrm{CH}=\mathrm{CH}_{2}, \mathrm{H}-2, \mathrm{H}-5, \mathrm{H}-6\right), 4.10$ $\left(\mathrm{dd}, 1 \mathrm{H}, J_{5,6^{\prime}}=6.1 \mathrm{~Hz}, J_{6,6^{\prime}}=12.8 \mathrm{~Hz}, \mathrm{H}-6^{\prime}\right), 2.05$ (s, 3 $\mathrm{H}, \mathrm{COCH}_{3}$ ). Anal. calcd for $\mathrm{C}_{25} \mathrm{H}_{26} \mathrm{O}_{9}$ : C, 63.82; $\mathrm{H}$, 
5.57. Found: $\mathrm{C}, 63.61 ; \mathrm{H}, 5.53$. For 8: $[\alpha]_{\mathrm{D}}-17.5(c 0.5$, $\left.\mathrm{CHCl}_{3}\right) ;{ }^{1} \mathrm{H}$ NMR $\left(400 \mathrm{MHz}, \mathrm{CDCl}_{3}\right): \delta$ 7.96-7.33 (m, $10 \mathrm{H}, 2 \mathrm{BzH}), 5.96\left(\mathrm{~m}, 1 \mathrm{H}, \mathrm{CH}=\mathrm{CH}_{2}\right), 5.78-5.76(\mathrm{~m}$, $2 \mathrm{H}, \mathrm{H}-3,4), 5.48(\mathrm{dd}, 1 \mathrm{H}, \mathrm{H}-2), 5.36\left(\mathrm{dd}, 1 \mathrm{H},{ }^{2} J=1.3\right.$ $\left.\mathrm{Hz},{ }^{3} J_{\text {trans }}=17.1 \mathrm{~Hz}, \mathrm{CH}=\mathrm{CH}_{2}\right), 5.28\left(\mathrm{dd}, 1 \mathrm{H},{ }^{2} J=1.3\right.$ $\left.\mathrm{Hz},{ }^{3} J_{\text {cis }}=10.4 \mathrm{~Hz}, \mathrm{CH}=\mathrm{CH}_{2}\right), 4.99\left(\mathrm{~d}, 1 \mathrm{H}, J_{1,2}=1.7\right.$ $\mathrm{Hz}, \mathrm{H}-1)$, 4.37-4.09 (m, $\left.5 \mathrm{H}, \mathrm{CH}_{2} \mathrm{CH}=\mathrm{CH}_{2}, \mathrm{H}-5,6,6^{\prime}\right)$, 2.16, $2.07\left(2 \mathrm{~s}, 6 \mathrm{H}, 2 \mathrm{COCH}_{3}\right)$. Anal. calcd for $\mathrm{C}_{27} \mathrm{H}_{28} \mathrm{O}_{10}$ : C, 63.28; H, 5.51. Found: $\mathrm{C}, 63.03 ; \mathrm{H}, 5.47$.

\subsection{Allyl 3,4-di- $O$-benzoyl- $\alpha$-D-mannopyranoside 9}

A solution of $8(5.6 \mathrm{~g}, 10.9 \mathrm{mmol})$ in $\mathrm{MeOH}(160 \mathrm{~mL})$ containing $0.5 \% \mathrm{HCl}$ was stirred at $\mathrm{rt}$ for $12 \mathrm{~h}$, at the end of which time TLC (2:1 petroleum ether-EtOAc) indicated that the starting material had disappeared. The mixture was neutralized with $\mathrm{Et}_{3} \mathrm{~N}$ and then concentrated to dryness. The residue was partitioned between water and $\mathrm{CH}_{2} \mathrm{Cl}_{2}$, the organic layer was dried over $\mathrm{Na}_{2} \mathrm{SO}_{4}$ and concentrated to a syrup. Purification of the residue by flash chromatography (2:1 petroleum ether-EtOAc) gave 9 as a syrup $(4.4 \mathrm{~g}, 93 \%):[\alpha]_{\mathrm{D}}-18.6$ (c 2.0, $\left.\mathrm{CHCl}_{3}\right) ;{ }^{1} \mathrm{H}$ NMR $\left(\mathrm{CDCl}_{3}, 400 \mathrm{MHz}\right): \delta 8.00$ $7.32(\mathrm{~m}, 10 \mathrm{H}, 2 \mathrm{Ph} H)$, $5.97\left(\mathrm{~m}, 1 \mathrm{H}, \mathrm{CH}=\mathrm{CH}_{2}\right)$, 5.82-5.75 (m, $2 \mathrm{H}, \mathrm{H}-3,4), 5.39-5.27(\mathrm{~m}, 2 \mathrm{H}, \mathrm{CH}=$ $\left.\mathrm{CH}_{2}\right), 5.05\left(\mathrm{~d}, 1 \mathrm{H}, J_{1.2}=1.6 \mathrm{~Hz}, \mathrm{H}-1\right), 4.31-3.70(\mathrm{~m}, 6$ $\left.\mathrm{H}, \mathrm{CH}_{2} \mathrm{CH}=\mathrm{CH}_{2}, \mathrm{H}-2,5,6,6^{\prime}\right)$. Anal. calcd for $\mathrm{C}_{23} \mathrm{H}_{24} \mathrm{O}_{8}$ : C, 64.48; H, 5.65. Found: $\mathrm{C}, 64.23 ; \mathrm{H}, 5.68$.

\subsection{Allyl 2,3,4,6-tetra- $O$-benzoyl- $\alpha$-D-mannopyranosyl- $(1 \rightarrow 2)-3,4,6$-tri- $O$-benzoyl- $\alpha$-D-mannopyranoside 10}

A solution of allyl 3,4,6-tri- $O$-benzoyl- $\alpha$-D-mannopyranoside $5(2.90 \mathrm{~g}, 5.45 \mathrm{mmol})$ and 2,3,4,6-tetra- $O$-benzoyl- $\alpha$-D-mannopyranosyl trichloroacetimidate 3 (4.44 $\mathrm{g}, 6.00 \mathrm{mmol})$ in dry $\mathrm{CH}_{2} \mathrm{Cl}_{2}(60 \mathrm{~mL})$ was stirred with dried molecular sieves ( $4 \AA$, $2 \mathrm{~g}$ ) under $\mathrm{N}_{2}$ for $15 \mathrm{~min}$, and then TMSOTf $(105 \mu \mathrm{L}, 0.55 \mathrm{mmol})$ was added. After $1 \mathrm{~h}$, the reaction mixture was neutralized with $\mathrm{Et}_{3} \mathrm{~N}$, filtered and the filtrate was concentrated. Purification of the residue by flash chromatography (3:1 petroleum ether-EtOAc) gave $\mathbf{1 0}$ as a syrup (5.33 g, $88 \%):[\alpha]_{\mathrm{D}}-37.9\left(c 1.0, \mathrm{CHCl}_{3}\right) ;{ }^{1} \mathrm{H}$ NMR $(400 \mathrm{MHz}$, $\left.\mathrm{CDCl}_{3}\right): \delta 8.10-7.20(\mathrm{~m}, 35 \mathrm{H}, 5 \mathrm{BzH}), 6.14-5.86(\mathrm{~m}, 6$ $\left.\mathrm{H}, \mathrm{CH}=\mathrm{CH}_{2}, 2 \mathrm{H}-4,2 \mathrm{H}-3, \mathrm{H}-2\right), 5.31(\mathrm{dd}, 1 \mathrm{H}$, $\left.{ }^{2} J=1.4 \mathrm{~Hz},{ }^{3} J_{\text {trans }}=18.5 \mathrm{~Hz}, \mathrm{CH}=\mathrm{CH}_{2}\right), 5.22(\mathrm{dd}, 1 \mathrm{H}$, $\left.{ }^{2} J=1.3 \mathrm{~Hz},{ }^{3} J_{\text {cis }}=10.4 \mathrm{~Hz}, \mathrm{CH}=\mathrm{CH}_{2}\right), 5.28(\mathrm{~d}, 1 \mathrm{H}$, $\left.J_{1,2}=1.6 \mathrm{~Hz}, \mathrm{H}-1\right), 5.22\left(\mathrm{~d}, 1 \mathrm{H}, J_{1,2}=1.6 \mathrm{~Hz}, \mathrm{H}-1\right)$, 4.69-4.40 (m, 7 H, H-2, $2 \mathrm{H}-5,4 \mathrm{H}-6)$, 4.27-3.99 (m, 2 $\mathrm{H}, \mathrm{CH}_{2} \mathrm{CH}=\mathrm{CH}_{2}$ ). Anal. calcd for $\mathrm{C}_{64} \mathrm{H}_{54} \mathrm{O}_{18}$ : C, 69.18; H, 4.90. Found: C, 68.98; H, 4.99.

\subsection{2,3,4,6-Tetra- $O$-benzoyl- $\alpha$-D-mannopyranosyl-( $1 \rightarrow$ 2)-3,4,6-tri- $O$-benzoyl- $\alpha$-D-mannopyranosyl trichloro- acetimidate 11}

A mixture of compound $10(3.82 \mathrm{~g}, 3.44 \mathrm{mmol})$ and $\mathrm{PdCl}_{2}(100 \mathrm{mg})$ in $\mathrm{MeOH}(150 \mathrm{~mL})$ was stirred vigorously for $4 \mathrm{~h}$ at $\mathrm{rt}$, TLC (2:1 petroleum ether-EtOAc) indicated that the reaction was complete. The reaction mixture was filtered through Celite and the filtrate was concentrated to dryness. The resulting compound was dissolved in $\mathrm{CH}_{2} \mathrm{Cl}_{2}(40 \mathrm{~mL})$, then $\mathrm{CCl}_{3} \mathrm{CN}(1.5 \mathrm{ml}, 15$ mmol) and DBU (100 $\mu \mathrm{L}, 0.71 \mathrm{mmol})$ were added. The reaction mixture was stirred for $2 \mathrm{~h}$, at the end of which time TLC (2:1 petroleum ether-EtOAc) indicated that the reaction was complete. Concentration of the reaction mixture followed by purification on a silica gel column with 2:1 petroleum ether-EtOAc as the eluent furnished the disaccharide donor 11 (3.34 g, 80\% two steps): $[\alpha]_{\mathrm{D}}-24.5\left(c 1.0, \mathrm{CHCl}_{3}\right) ;{ }^{1} \mathrm{H}$ NMR $(400 \mathrm{MHz}$, $\left.\mathrm{CDCl}_{3}\right): \delta 8.72(\mathrm{~s}, 1 \mathrm{H}, \mathrm{NH}), 8.09-7.26(\mathrm{~m}, 35 \mathrm{H}, 7$ $\mathrm{Bz} H), 6.64\left(\mathrm{~d}, 1 \mathrm{H}, J_{2,1}=1.9 \mathrm{~Hz}, \mathrm{H}-1\right), 6.19-5.93(\mathrm{~m}, 5$ $\mathrm{H}, 2 \mathrm{H}-4,2 \mathrm{H}-3,1 \mathrm{H}-2), 5.37\left(\mathrm{~d}, 1 \mathrm{H}, J_{1^{\prime}, 2^{\prime}}=1.5 \mathrm{~Hz}\right.$, $\mathrm{H}-1$ ), 4.75-4.48 (m, $7 \mathrm{H}, \mathrm{H}-2,2 \mathrm{H}-5,4 \mathrm{H}-6)$. Anal. calcd for $\mathrm{C}_{63} \mathrm{H}_{50} \mathrm{Cl}_{3} \mathrm{NO}_{18}$ : C, 62.26; H, 4.15. Found: $\mathrm{C}$, 62.08; H, 4.19.

\subsection{Allyl 2,3,4,6-tetra- $O$-benzoyl- $\alpha$-D-mannopyranosyl- $(1 \rightarrow 2)-6-O$-acetyl-3,4-di- $O$-benzoyl- $\alpha$-D- mannopyranoside 12}

A solution of $7(1.5 \mathrm{~g}, 3.19 \mathrm{mmol})$ and 2,3,4,6-tetra- $O$ benzoyl- $\alpha$-D-mannopyranosyl trichloroacetimidate (3) (2.60 g, $3.51 \mathrm{mmol})$ in dry $\mathrm{CH}_{2} \mathrm{Cl}_{2}(40 \mathrm{~mL})$ was stirred with dried molecular sieves $(4 \AA, 1 \mathrm{~g})$ under $\mathrm{N}_{2}$ for 15 min, and then TMSOTf $(50 \mu \mathrm{L})$ was added. After $1 \mathrm{~h}$, the reaction mixture was neutralized with $\mathrm{Et}_{3} \mathrm{~N}$, filtered and the filtrate was concentrated. Purification of the residue by flash chromatography (3:1 petroleum etherEtOAc) gave 12 as a syrup $(3.35 \mathrm{~g}, 92 \%):[\alpha]_{\mathrm{D}}-45.3(c$ 1.0, $\left.\mathrm{CHCl}_{3}\right) ;{ }^{1} \mathrm{H}$ NMR (400 MHz, $\left.\mathrm{CDCl}_{3}\right): \delta 8.01-7.26$ $(\mathrm{m}, 30 \mathrm{H}, 6 \mathrm{Bz} H), 6.10-5.86\left(\mathrm{~m}, 6 \mathrm{H}, \mathrm{CH}=\mathrm{CH}_{2}, 2 \mathrm{H}-4\right.$, $2 \mathrm{H}-3, \mathrm{H}-2), 5.35-5.31\left(\mathrm{dd}, 1 \mathrm{H},{ }^{2} J=1.5 \mathrm{~Hz},{ }^{3} J_{\text {trans }}=\right.$ $\left.17.2 \mathrm{~Hz}, \mathrm{CH}=\mathrm{CH}_{2}\right), 5.27-5.24\left(\mathrm{dd}, 1 \mathrm{H},{ }^{2} \mathrm{~J}=1.3 \mathrm{~Hz}\right.$, $\left.{ }^{3} J_{\text {cis }}=10.4 \mathrm{~Hz}, \mathrm{CH}=\mathrm{CH}_{2}\right), 5.25\left(\mathrm{~d}, 1 \mathrm{H}, J_{2,1}=1.7 \mathrm{~Hz}\right.$, $\mathrm{H}-1), 5.21\left(\mathrm{~d}, 1 \mathrm{H}, J_{2,1}=1.7 \mathrm{~Hz}, \mathrm{H}-1\right), 4.72-4.01(\mathrm{~m}, 9$ $\left.\mathrm{H}, \mathrm{CH} \mathrm{CH}_{2} \mathrm{CH} \mathrm{CH}_{2}, \mathrm{H}-2,2 \mathrm{H}-5,4 \mathrm{H}-6\right), 2.17$ (s, $3 \mathrm{H}$, $\mathrm{COCH}_{3}$ ). Anal. calcd for $\mathrm{C}_{59} \mathrm{H}_{52} \mathrm{O}_{18}: \mathrm{C}, 67.55 ; \mathrm{H}, 5.00$. Found: C, 67.75; H, 5.04.

\section{8. ,3,4,6-Tetra- $O$-benzoyl- $\alpha$-D-mannopyranosyl-( $(1 \rightarrow 2)$ - 6- $O$-acetyl-3,4-di- $O$-benzoyl- $\alpha$-D-mannopyranosyl trichloroacetimidate 13}

A mixture of compound $\mathbf{1 2}(2.30 \mathrm{~g}, 2.19 \mathrm{mmol})$ and $\mathrm{PdCl}_{2}(70 \mathrm{mg})$ in $\mathrm{MeOH}(100 \mathrm{~mL})$ was stirred vigorously for $4 \mathrm{~h}$ at rt, TLC (2:1 petroleum ether-EtOAc) indicated that the reaction was complete. The reaction mixture was filtered through Celite, and the filtrate was concentrated to dryness. The resulting compound was dissolved in $\mathrm{CH}_{2} \mathrm{Cl}_{2}(30 \mathrm{~mL})$, then $\mathrm{CCl}_{3} \mathrm{CN}(1.0 \mathrm{~mL}, 10$ $\mathrm{mmol})$ and $\mathrm{DBU}(50 \mu \mathrm{L})$ were added. The reaction mixture was stirred for $2 \mathrm{~h}$, at the end of which time TLC (2:1 petroleum ether-EtOAc) indicated that the reaction was complete. Concentration of the reaction mixture followed by purification on a silica gel column with 2:1 petroleum ether-EtOAc as the eluent, furnished the disaccharide donor $13(2.05 \mathrm{~g}, 81 \%$ two steps): $[\alpha]_{\mathrm{D}}-43.7\left(c 1.0, \mathrm{CHCl}_{3}\right) ;{ }^{1} \mathrm{H}$ NMR $(400 \mathrm{MHz}$, $\left.\mathrm{CDCl}_{3}\right): \delta 8.73(\mathrm{~s}, 1 \mathrm{H}, \mathrm{NH}), 8.09-7.26(\mathrm{~m}, 30 \mathrm{H}, 6$ $\mathrm{BzH}), 6.65\left(\mathrm{~d}, 1 \mathrm{H}, J_{2,1}=1.7 \mathrm{~Hz}, \mathrm{H}-1\right), 6.19-5.89(\mathrm{~m}, 5$ $\mathrm{H}, 2 \mathrm{H}-4,2 \mathrm{H}-3, \mathrm{H}-2), 5.35\left(\mathrm{~d}, 1 \mathrm{H}, J_{2^{\prime}, 1^{\prime}}=1.3 \mathrm{~Hz}, \mathrm{H}-1\right)$, 4.80-4.34 (m, 7 H, H-2, 2 H-5, 4 H-6), 2.17 (s, 3 H, 
$\mathrm{COCH}_{3}$ ). Anal. calcd for $\mathrm{C}_{58} \mathrm{H}_{48} \mathrm{Cl}_{3} \mathrm{NO}_{18}: \mathrm{C}, 60.40 ; \mathrm{H}$, 4.19. Found: C, 60.61; H, 4.16.

\section{9. $2,3,4,6$-Tetra- $O$-benzoyl- $\alpha$-D-mannopyranosyl-( $1 \rightarrow$ 2)-6- $O$-acetyl-3,4-di- $O$-benzoyl- $\alpha$-D-mannopyranosyl-(1 $\rightarrow 6$ )-3,4-di- $O$-benzoyl- $\alpha$-D-mannopyranoside 14}

A solution of $13(1.76 \mathrm{~g}, 1.53 \mathrm{mmol})$ and 3,4-di- $O$-benzoyl- $\alpha$-D-mannopyranoside $9(647 \mathrm{mg}, 1.51 \mathrm{mmol})$ in dry $\mathrm{CH}_{2} \mathrm{Cl}_{2}(30 \mathrm{~mL})$ was stirred with dried molecular sieves $(4 \mathrm{~A}, 1 \mathrm{~g})$ under $\mathrm{N}_{2}$ for $15 \mathrm{~min}$, and then TMSOTf $(30 \mu \mathrm{L})$ was added. After $1 \mathrm{~h}$, the reaction mixture was neutralized with $\mathrm{Et}_{3} \mathrm{~N}$, filtered and the filtrate was concentrated. Purification of the residue by column chromatography (2:1 petroleum ether-EtOAc) gave 14 as a syrup $(1.96 \mathrm{~g}, 91 \%):[\alpha]_{\mathrm{D}}-33.2(c 1.0$, $\left.\mathrm{CHCl}_{3}\right) ;{ }^{1} \mathrm{H}$ NMR $\left(400 \mathrm{MHz}, \mathrm{CDCl}_{3}\right): \delta 8.08-7.32(\mathrm{~m}$, $40 \mathrm{H}, 8 \mathrm{BzH}), 6.13-5.72\left(\mathrm{~m}, 8 \mathrm{H}, \mathrm{CH}=\mathrm{CH}_{2}, \mathrm{H}-2,3\right.$ H-3, $3 \mathrm{H}-4), 5.44\left(\mathrm{dd}, 1 \mathrm{H},{ }^{2} J=1.5 \mathrm{~Hz},{ }^{3} J_{\text {trans }}=17.2 \mathrm{~Hz}\right.$, $\left.\mathrm{CH}=\mathrm{CH}_{2}\right), 5.28\left(\mathrm{dd}, 1 \mathrm{H},{ }^{2} J=1.3 \mathrm{~Hz},{ }^{3} J_{\text {cis }}=10.5 \mathrm{~Hz}\right.$, $\left.\mathrm{CH}=\mathrm{CH}_{2}\right), 5.29\left(\mathrm{~d}, 1 \mathrm{H}, J_{1,2}=1.5 \mathrm{~Hz}, \mathrm{H}-1\right), 5.23(\mathrm{~d}, 1$ $\left.\mathrm{H}, J_{1,2}=1.5 \mathrm{~Hz}, \mathrm{H}-1\right), 5.05\left(\mathrm{~d}, 1 \mathrm{H}, J_{1,2}=1.5 \mathrm{~Hz}, \mathrm{H}-1\right)$, $4.76(\mathrm{dd}, 1 \mathrm{H}, \mathrm{H}-6), 4.65$ (dd, $1 \mathrm{H}, \mathrm{H}-6), 4.46-4.10$ (m, $\left.9 \mathrm{H}, \mathrm{CH}_{2} \mathrm{CH}=\mathrm{CH}_{2}, 3 \mathrm{H}-5,2 \mathrm{H}-6,2 \mathrm{H}-2\right), 3.90$ (dd, 1 $\mathrm{H}, \mathrm{H}-6), 3.71$ (dd, $1 \mathrm{H}, \mathrm{H}-6), 2.11$ (s, $\left.3 \mathrm{H}, \mathrm{COCH}_{3}\right)$. Anal. calcd for $\mathrm{C}_{79} \mathrm{H}_{70} \mathrm{O}_{25}: \mathrm{C}, 66.85 ; \mathrm{H}, 4.97$; Found: $\mathrm{C}$, 67.07; H, 4.92.

4.10. $2,3,4,6$-Tetra- $O$-benzoyl- $\alpha$-D-mannopyranosyl-( $1 \rightarrow$ 2)-6- $O$-acetyl-3,4-di- $O$-benzoyl- $\alpha$-D-mannopyranosyl-(1 $\rightarrow 6)-2-O$-acetyl-3,4-di- $O$-benzoyl- $\alpha$-D-mannopyranoside 15

To a solution of $\mathbf{1 4}(1.20 \mathrm{~g}, 0.84 \mathrm{mmol})$ in pyridine (20 $\mathrm{mL}) \mathrm{Ac}_{2} \mathrm{O}(2 \mathrm{~mL})$ was added. After the mixture was stirred for $3 \mathrm{~h}$ at rt, it was diluted with $\mathrm{CH}_{2} \mathrm{Cl}_{2}$, washed with $1 \mathrm{~N} \mathrm{HCl}$, water, and satd aq $\mathrm{NaHCO}_{3}$. The organic layers were combined, dried, and concentrated. Purification by column chromatography $(2: 1$ petroleum ether-EtOAc) quantitatively gave $\mathbf{1 5}$ as a syrup: $[\alpha]_{\mathrm{D}}$ -29.3 (c 1.5, $\left.\mathrm{CHCl}_{3}\right) ;{ }^{1} \mathrm{H}$ NMR (400 MHz, $\left.\mathrm{CDCl}_{3}\right): \delta$ 8.03-7.27 (m, 40 H, 8 BzH), 6.14-5.79 (m, 8 H, CH= $\left.\mathrm{CH}_{2}, \mathrm{H}-2,3 \mathrm{H}-3,3 \mathrm{H}-4\right), 5.53(\mathrm{dd}, 1 \mathrm{H}, \mathrm{H}-2), 5.46$ (dd, $\left.1 \mathrm{H},{ }^{2} J=1.5 \mathrm{~Hz},{ }^{3} J_{\text {trans }}=17.2 \mathrm{~Hz}, \mathrm{CH}=\mathrm{CH}_{2}\right), 5.31(\mathrm{dd}$, $\left.1 \mathrm{H},{ }^{2} \mathrm{~J}=1.3 \mathrm{~Hz},{ }^{3} J_{\text {cis }}=10.5 \mathrm{~Hz}, \mathrm{CH}=\mathrm{CH}_{2}\right), 5.23(\mathrm{~d}, 1$ $\left.\mathrm{H}, J_{1,2}=1.5 \mathrm{~Hz}, \mathrm{H}-1\right), 5.20\left(\mathrm{~d}, 1 \mathrm{H}, J_{1,2}=1.5 \mathrm{~Hz}, \mathrm{H}-1\right)$, $5.02\left(\mathrm{~d}, 1 \mathrm{H}, J_{1.2}=1.5 \mathrm{~Hz}, \mathrm{H}-1\right), 4.73(\mathrm{dd}, 1 \mathrm{H}, \mathrm{H}-6)$, 4.62 (dd, $1 \mathrm{H}, \mathrm{H}-6), 4.47-4.09\left(\mathrm{~m}, 8 \mathrm{H}, \mathrm{CH}_{2} \mathrm{CH}=\mathrm{CH}_{2}\right.$, $3 \mathrm{H}-5,2 \mathrm{H}-6, \mathrm{H}-2), 3.92$ (dd, $1 \mathrm{H}, \mathrm{H}-6), 3.65$ (dd, $1 \mathrm{H}$, $\mathrm{H}-6), 2.19,2.08\left(2 \mathrm{~s}, 6 \mathrm{H}, 2 \mathrm{COCH}_{3}\right)$. Anal. calcd for $\mathrm{C}_{81} \mathrm{H}_{72} \mathrm{O}_{26}$ : C, 66.57; H, 4.97; Found: C, 66.34; H, 5.01.

4.11. Allyl $2,3,4,6$-tetra- $O$-benzoyl- $\alpha$-D-mannopyranosyl$(1 \rightarrow 2)-6-O$-acetyl-3,4-di- $O$-benzoyl- $\alpha$-D-mannopyranosyl-(1 $\rightarrow 6)-[2,3,4,6$-tetra- $O$-benzoyl- $\alpha$-D-mannopyranosyl$(1 \rightarrow 2)-3,4,6$-tri- $O$-benzoyl- $\alpha$-D-mannopyranosyl-( $1 \rightarrow 2)]-$ 3,4-di- $O$-benzoyl- $\alpha$-D-mannopyranoside 16

A solution of $14(1.65 \mathrm{~g}, 1.16 \mathrm{mmol})$ and $11(1.56 \mathrm{~g}$, $1.28 \mathrm{mmol})$ in dry $\mathrm{CH}_{2} \mathrm{Cl}_{2}(30 \mathrm{~mL})$ was stirred with dried molecular sieves ( $4 \AA, 1.5 \mathrm{~g})$ under $\mathrm{N}_{2}$ for $15 \mathrm{~min}$, and then TMSOTf $(20 \mu \mathrm{L})$ was added. After $1 \mathrm{~h}$, the reaction mixture was neutralized with $\mathrm{Et}_{3} \mathrm{~N}$, filtered and the filtrate was concentrated. Purification of the residue by column chromatography $(1.5: 1$ petroleum etherEtOAc) gave 16 as a syrup $(2.87 \mathrm{~g}, 90 \%):[\alpha]_{\mathrm{D}}-45.2(c$ 1.0, $\left.\mathrm{CHCl}_{3}\right) ;{ }^{1} \mathrm{H}$ NMR (400 MHz, $\left.\mathrm{CDCl}_{3}\right): \delta 8.00-6.93$ $(\mathrm{m}, 75 \mathrm{H}, 15 \mathrm{BzH}), 6.20-5.75\left(\mathrm{~m}, 13 \mathrm{H}, \mathrm{CH}=\mathrm{CH}_{2}, 5\right.$ $\mathrm{H}-4,5 \mathrm{H}-3,2 \mathrm{H}-2), 5.60\left(\mathrm{~d}, 1 \mathrm{H}, J_{1,2}=1.6 \mathrm{~Hz}, \mathrm{H}-1\right)$, $5.58\left(\mathrm{~d}, 1 \mathrm{H}, J_{1.2}=1.6 \mathrm{~Hz}, \mathrm{H}-1\right), 5.41\left(\mathrm{dd}, 1 \mathrm{H},{ }^{2} J=1.5\right.$ $\left.\mathrm{Hz},{ }^{3} J_{\text {trans }}=18.7 \mathrm{~Hz}, \mathrm{CH}=\mathrm{CH}_{2}\right), 5.39\left(\mathrm{~d}, 1 \mathrm{H}, J_{1,2}=1.6\right.$ $\mathrm{Hz}, \mathrm{H}-1), 5.28\left(\mathrm{dd}, 1 \mathrm{H},{ }^{2} J=1.3 \mathrm{~Hz},{ }^{3} J_{\text {cis }}=11.8 \mathrm{~Hz}\right.$, $\left.\mathrm{CH}=\mathrm{CH}_{2}\right), 5.27\left(\mathrm{~d}, 1 \mathrm{H}, J_{1,2}=1.6 \mathrm{~Hz}, \mathrm{H}-1\right), 5.12(\mathrm{~d}, 1$ $\left.\mathrm{H}, J_{1,2}=1.6 \mathrm{~Hz}, \mathrm{H}-1\right), 1.95\left(\mathrm{~s}, 3 \mathrm{H}, \mathrm{COCH}_{3}\right) .{ }^{13} \mathrm{C} \mathrm{NMR}$ $\left(100 \mathrm{MHz}, \mathrm{CDCl}_{3}\right): \delta 170.66\left(1 \mathrm{C}, \mathrm{COCH}_{3}\right), 166.17$, $166.03,165.70,165.59,165.59,165.59,165.44,165.19$, $165.19,165.19,165.13,164.94,164.94,164.66,164.65$ (15 C, $15 \mathrm{COPh}$ ), 133.4-128.0 (91 C, $15 \mathrm{Ph}, \mathrm{CH}_{2} \mathrm{CH}=$ $\left.\mathrm{CH}_{2}\right), 117.80\left(\mathrm{CH}_{2} \mathrm{CH}=\mathrm{CH}_{2}\right), \quad 100.04,99.65,99.42$, 98.36, $98.04\left(5 \mathrm{C}-1,{ }^{2} J_{\mathrm{Cl}-\mathrm{H} 1}=170-176.8 \mathrm{~Hz}\right), 78.95$, 77.66, 77.20 (3 C-2), $20.5\left(1 \mathrm{C}, \mathrm{COCH}_{3}\right)$. Anal. calcd for $\mathrm{C}_{140} \mathrm{H}_{118} \mathrm{O}_{42}: \mathrm{C}, 68.01 ; \mathrm{H}, 4.81$. Found: $\mathrm{C}, 68.29 ; \mathrm{H}$, 4.77 .

\subsection{A one-pot procedure for the preparation of the pentasaccharide 16}

A solution of $9(0.9 \mathrm{~g}, 2.10 \mathrm{mmol})$ and $13(2.45 \mathrm{~g}, 2.13$ $\mathrm{mmol})$ in dry $\mathrm{CH}_{2} \mathrm{Cl}_{2}(100 \mathrm{~mL})$ was stirred with activated $4 \AA$ molecular sieves $(2 \mathrm{~g})$ at $\mathrm{rt}$ under $\mathrm{N}_{2}$ for 20 $\min$. Then the reaction mixture was cooled to $-45^{\circ} \mathrm{C}$, and TMSOTf $(10 \mu \mathrm{L})$ was added. After $30 \mathrm{~min}$, temperature was allowed to rise to rt. After the reaction mixture was stirred for further $1 \mathrm{~h}$, to the reaction mixture was added 11 (2.83 g, $2.33 \mathrm{mmol})$ under $\mathrm{N}_{2}$ at rt. The reaction mixture was stirred for $1 \mathrm{~h}$, at the end of which time TLC (2:1 petroleum ether-EtOAc) indicated that the reaction was complete. The reaction mixture was neutralized with $\mathrm{Et}_{3} \mathrm{~N}$, filtered and the filtrate was concentrated. Purification of the resultant residue by column chromatography with $2: 1$ petroleum ether-EtOAc as eluent gave $\mathbf{1 6}(4.7 \mathrm{~g}, 83 \%)$.

4.13. $2,3,4,6$-Tetra- $O$-benzoyl- $\alpha$-D-mannopyranosyl-( $1 \rightarrow$ 2)-6- $O$-acetyl-3,4-di- $O$-benzoyl- $\alpha$-D-mannopyranosyl-(1 $\rightarrow 6)-[2,3,4,6$-tetra- $O$-benzoyl- $\alpha$-D-mannopyranosyl- $(1 \rightarrow$ 2)-3,4,6-tri- $O$-benzoyl- $\alpha$-D-mannopyranosyl- $(1 \rightarrow 2) \mid-3,4-$ di- $O$-benzoyl- $\alpha$-D-mannopyranosyl trichloroacetimidate 17

A mixture of compound $\mathbf{1 6}(1.00 \mathrm{~g}, 0.36 \mathrm{mmol})$ and $\mathrm{PdCl}_{2}(30 \mathrm{mg})$ in $\mathrm{MeOH}(50 \mathrm{~mL})$ was stirred vigorously for $4 \mathrm{~h}$ at $\mathrm{rt}$, TLC (1.5:1 petroleum ether-EtOAc) indicated that the reaction was complete. The reaction mixture was filtered through Celite and the filtrate was concentrated to dryness. The resulting compound was dissolved in $\mathrm{CH}_{2} \mathrm{Cl}_{2}(15 \mathrm{~mL})$, then $\mathrm{CCl}_{3} \mathrm{CN}(0.5 \mathrm{ml}, 5$ $\mathrm{mmol})$ and DBU $(50 \mu \mathrm{L})$ were added. The reaction mixture was stirred for $2 \mathrm{~h}$, at the end of which time TLC (2:1 petroleum ether-EtOAc) indicated that the reaction was complete. Concentration of the reaction mixture followed by purification on a silica gel column with 2:1 petroleum ether-EtOAc as the eluent furnished 
the pentasaccharide donor $17(798 \mathrm{mg}, 85 \%$ two steps): $[\alpha]_{\mathrm{D}}-35.0 \quad\left(c \quad 1.0, \mathrm{CHCl}_{3}\right) ;{ }^{1} \mathrm{H}$ NMR $(400$ $\left.\mathrm{MHz}, \mathrm{CDCl}_{3}\right): \delta 8.76(\mathrm{~s}, 1 \mathrm{H}, \mathrm{NH}), 8.05-6.81(\mathrm{~m}, 75$ $\mathrm{H}, 15 \mathrm{Bz} H), 6.55\left(\mathrm{~d}, 1 \mathrm{H}, J_{1,2} 1.6 \mathrm{~Hz}, \mathrm{H}-1\right), 6.16$ $5.87(\mathrm{~m}, 12 \mathrm{H}, 5 \mathrm{H}-4,5 \mathrm{H}-3,2 \mathrm{H}-2), 5.70(\mathrm{~d}, 1 \mathrm{H}$, $\left.J_{1,2}=1.3 \mathrm{~Hz}, \mathrm{H}-1\right), 5.63\left(\mathrm{~d}, 1 \mathrm{H}, J_{1,2}=1.3 \mathrm{~Hz}, \mathrm{H}-1\right)$, $5.38\left(\mathrm{~d}, 1 \mathrm{H}, J_{1,2}=1.3 \mathrm{~Hz}, \mathrm{H}-1\right), 5.36\left(\mathrm{~d}, 1 \mathrm{H}, J_{1,2}=\right.$ $1.3 \mathrm{~Hz}, \mathrm{H}-1), 4.99-3.60(\mathrm{~m}, 18 \mathrm{H}, 3 \mathrm{H}-2,5 \mathrm{H}-5,10$ $\mathrm{H}-6), 1.93\left(\mathrm{~s}, 3 \mathrm{H}, \mathrm{COCH}_{3}\right)$. Anal. calcd for $\mathrm{C}_{139} \mathrm{H}_{114} \mathrm{Cl}_{3} \mathrm{NO}_{42}: \mathrm{C}$, 64.79; $\mathrm{H}, 4.46$. Found: $\mathrm{C}$, 64.56; $\mathrm{H}, 4.50$.

4.14. Allyl 2,3,4,6-tetra- $O$-benzoyl- $\alpha$-D-mannopyranosyl$(1 \rightarrow 2)$-3,4-di- $O$-benzoyl- $\alpha$-D-mannopyranosyl- $(1 \rightarrow 6)$ $[2,3,4,6$-tetra- $O$-benzoyl- $\alpha$-D-mannopyranosyl-( $1 \rightarrow 2)$ 3,4,6-tri- $O$-benzoyl- $\alpha$-D-mannopyranosyl- $(1 \rightarrow 2)]-$ 3,4-di- $O$-benzoyl- $\alpha$-D-mannopyranoside 18

A solution of $16(1.2 \mathrm{~g}, 0.44 \mathrm{mmol})$ in $\mathrm{MeOH}(100$ $\mathrm{mL}$ ) containing $0.5 \% \mathrm{HCl}$ was stirred at $\mathrm{rt}$ for $12 \mathrm{~h}$, at the end of which time TLC (1.5:1 petroleum etherEtOAc) indicated that the starting material had disappeared. The mixture was neutralized with $\mathrm{Et}_{3} \mathrm{~N}$, and then concentrated to dryness. The residue was partitioned between water and $\mathrm{CH}_{2} \mathrm{Cl}_{2}$, the organic layer was dried over $\mathrm{Na}_{2} \mathrm{SO}_{4}$, and concentrated to a syrup. Purification of the residue by column chromatography (1.5:1 petroleum ether-EtOAc) gave $\mathbf{1 8}$ as a syrup (978 mg, 92\%): $\left.[\alpha]_{\mathrm{D}}-62.7(c) 1.0, \mathrm{CHCl}_{3}\right) ;{ }^{1} \mathrm{H}$ NMR $\left(400 \mathrm{MHz}, \mathrm{CDCl}_{3}\right): \delta 8.03-6.96(\mathrm{~m}, 75 \mathrm{H}, 15 \mathrm{BzH})$, 6.13-5.71 (m, $\left.13 \mathrm{H}, \mathrm{CH}=\mathrm{CH}_{2}, 5 \mathrm{H}-4,5 \mathrm{H}-3,2 \mathrm{H}-2\right)$, $5.56\left(\mathrm{~d}, 1 \mathrm{H}, J_{1,2}=1.2 \mathrm{~Hz}, \mathrm{H}-1\right), 5.51\left(\mathrm{~d}, 1 \mathrm{H}, J_{1,2}=\right.$ $1.2 \mathrm{~Hz}, \mathrm{H}-1), 5.40\left(\mathrm{dd}, 1 \mathrm{H},{ }^{2} J=1.5 \mathrm{~Hz},{ }^{3} J_{\text {trans }}=17.2\right.$ $\left.\mathrm{Hz}, \mathrm{CH}=\mathrm{CH}_{2}\right), 5.31\left(\mathrm{~d}, 1 \mathrm{H}, J_{1,2}=1.2 \mathrm{~Hz}, \mathrm{H}-1\right), 5.29$ $\left(\mathrm{dd}, 1 \mathrm{H},{ }^{2} J=1.3 \mathrm{~Hz},{ }^{3} J_{\text {cis }}=10.4 \mathrm{~Hz}\right), 5.25(\mathrm{~d}, 1 \mathrm{H}$, $\left.J_{1,2}=1.2 \mathrm{~Hz}, \mathrm{H}-1\right), 5.08\left(\mathrm{~d}, 1 \mathrm{H}, J_{1,2}=1.2 \mathrm{~Hz}, \mathrm{H}-1\right)$, 4.86-3.41 (m, $20 \mathrm{H}, \mathrm{CH}_{2} \mathrm{CH}=\mathrm{CH}_{2}, 3 \mathrm{H}-2,5 \mathrm{H}-5,10$ $\mathrm{H}-6)$. Anal. calcd for $\mathrm{C}_{138} \mathrm{H}_{116} \mathrm{O}_{41}$ : C, 68.20; H, 4.81. Found: C, 68.41; H, 4.71.

4.15. Allyl 2,3,4,6-tetra- $O$-benzoyl- $\alpha$-D-mannopyranosyl$(1 \rightarrow 2)$-6- $O$-acetyl-3,4-di- $O$-benzoyl- $\alpha$-D-mannopyranosyl- $(1 \rightarrow 6)-[2,3,4,6$-tetra- $O$-benzoyl- $\alpha$-D-mannopyranosyl$(1 \rightarrow 2)-3,4,6$-tri- $O$-benzoyl- $\alpha$-D-mannopyranosyl- $(1 \rightarrow 2)$ 3,4-di- $O$-benzoyl- $\alpha$-D-mannopyranosyl- $(1 \rightarrow 6)-[2,3,4,6-$ tetra- $O$-benzoyl- $\alpha$-D-mannopyranosyl- $(1 \rightarrow 2) \mid-3,4-d i-O$ benzoyl- $\alpha$-D-mannopyranosyl- $(1 \rightarrow 6)-[2,3,4,6$-tetra- $O$ benzoyl- $\alpha$-D-mannopyranosyl- $(1 \rightarrow 2)-3,4,6$-tri- $O$ benzoyl- $\alpha$-D-mannopyranosyl-( $1 \rightarrow 2)$ ]-3,4-di- $O$-benzoyl$\alpha$-D-mannopyranoside 19

A solution of $17(550 \mathrm{mg}, 0.21 \mathrm{mmol})$ and 18 (461 $\mathrm{mg}, 0.19 \mathrm{mmol})$ in dry $\mathrm{CH}_{2} \mathrm{Cl}_{2}(20 \mathrm{~mL})$ was stirred with dried molecular sieves $(4 \mathrm{~A}, 0.5 \mathrm{~g})$ under $\mathrm{N}_{2}$ for $15 \mathrm{~min}$, and then TMSOTf $(5 \mu \mathrm{L})$ was added. After 1 $\mathrm{h}$, the reaction mixture was neutralized with $\mathrm{Et}_{3} \mathrm{~N}$, filtered and the filtrate was concentrated. Purification of the residue by a column chromatography (1.3:1 petroleum ether-EtOAc) gave 19 as a syrup (460 mg, $50 \%):[\alpha]_{\mathrm{D}}-33.7\left(c 1.0, \mathrm{CHCl}_{3}\right) ;{ }^{1} \mathrm{H}$ NMR $(400 \mathrm{MHz}$, $\left.\mathrm{CDCl}_{3}\right): \delta 8.02-6.60(\mathrm{~m}, 150 \mathrm{H}, 30 \mathrm{BzH}), 6.40-5.82$ $\left(\mathrm{m}, 25 \mathrm{H}, \mathrm{CH}=\mathrm{CH}_{2} 10 \mathrm{H}-4,10 \mathrm{H}-3,4 \mathrm{H}-2\right), 5.70(\mathrm{~d}$, $\left.1 \mathrm{H}, J_{1,2}=1.3 \mathrm{~Hz}, \mathrm{H}-1\right), 5.62(\mathrm{~m}, 2 \mathrm{H}, 2 \mathrm{H}-1), 5.48$ $\left(\mathrm{d}, 1 \mathrm{H}, J_{1,2}=1.3 \mathrm{~Hz}, \mathrm{H}-1\right), 5.39\left(\mathrm{~d}, 1 \mathrm{H}, J_{1,2}=1.3 \mathrm{~Hz}\right.$, $\mathrm{H}-1), 5.34\left(\mathrm{dd}, 1 \mathrm{H},{ }^{2} J=1.3 \mathrm{~Hz},{ }^{3} J_{\text {trans }}=17.1 \mathrm{~Hz}\right.$, $\left.\mathrm{CH}=\mathrm{CH}_{2}\right), 5.32\left(\mathrm{~d}, 1 \mathrm{H}, J_{1.2}=1.3 \mathrm{~Hz}, \mathrm{H}-1\right), 5.28(\mathrm{~d}$, $\left.1 \mathrm{H}, J_{1,2}=1.3 \mathrm{~Hz}, \mathrm{H}-1\right), 5.24\left(\mathrm{~d}, 1 \mathrm{H}, J_{1,2}=1.3 \mathrm{~Hz}\right.$, $\mathrm{H}-1), 5.22\left(\mathrm{dd}, 1 \mathrm{H},{ }^{2} J=1.3 \mathrm{~Hz},{ }^{3} J_{\text {cis }}=10.4 \mathrm{~Hz}, \mathrm{CH}=\right.$ $\left.\mathrm{CH}_{2}\right), 5.20\left(\mathrm{~d}, 1 \mathrm{H}, J_{1,2}=1.3 \mathrm{~Hz}, \mathrm{H}-1\right), 4.98(\mathrm{~d}, 1 \mathrm{H}$, $\left.J_{1,2}=1.3 \mathrm{~Hz}, \mathrm{H}-1\right), 4.93-3.26\left(\mathrm{~m}, 38 \mathrm{H}, \mathrm{CH}_{2} \mathrm{CH}=\right.$ $\left.\mathrm{CH}_{2}, 10 \mathrm{H}-5,20 \mathrm{H}-6.6 \mathrm{H}-2\right), 1.87\left(\mathrm{~s}, 3 \mathrm{H}, \mathrm{COCH}_{3}\right)$; ${ }^{13} \mathrm{C}$ NMR $\left(100 \mathrm{MHz}, \mathrm{CDCl}_{3}\right): \quad \delta \quad 170.69 \quad(1 \mathrm{C}$, $\left.\mathrm{COCH}_{3}\right), 166.2-164.5(30 \mathrm{C}, 30 \mathrm{COPh}), 133.4-127.5$ $\left(181 \mathrm{C}, 30 \mathrm{Ph}, \mathrm{CH}=\mathrm{CH}_{2}\right), 117.7\left(\mathrm{CH}=\mathrm{CH}_{2}\right), 100.07$, $99.99,99.72,99.55,99.43,99.34,98.89,98.26,98.19$, $98.17(10 \mathrm{C}-1), 20.4\left(1 \mathrm{C}, \mathrm{COCH}_{3}\right)$. Anal. calcd for $\mathrm{C}_{275} \mathrm{H}_{228} \mathrm{O}_{82}$ : C, 68.18; H, 4.74. Found: $\mathrm{C}, 68.32 ; \mathrm{H}$, 4.80 .

\subsection{Allyl $\alpha$-D-mannopyranosyl-( $1 \rightarrow 2)-\alpha$-D-manno- pyranosyl-( $1 \rightarrow 6)$-[ $\alpha$-D-mannopyranosyl- $(1 \rightarrow 2)-\alpha$-D- mannopyranosyl- $(1 \rightarrow 2) \mid-\alpha$-D-mannopyranoside 1}

Compound 16 (758 mg, $0.28 \mathrm{mmol})$ was dissolved in an ammonia-saturated solution of 1:9 $\mathrm{CH}_{2} \mathrm{Cl}_{2}-$ $\mathrm{CH}_{3} \mathrm{OH}(100 \mathrm{~mL})$ at $40^{\circ} \mathrm{C}$. After $24 \mathrm{~h}$, the reaction mixture was concentrated to about $10 \mathrm{~mL}$, and then $\mathrm{CH}_{2} \mathrm{Cl}_{2}(100 \mathrm{~mL})$ was added. The resultant precipitate was filtered and washed four times with $\mathrm{CH}_{2} \mathrm{Cl}_{2}$ to afford 1 as white solid $(219 \mathrm{mg}, 91 \%):[\alpha]_{\mathrm{D}}+12.3$ (c 1.0, $\left.\mathrm{H}_{2} \mathrm{O}\right) ;{ }^{1} \mathrm{H}$ NMR $\left(\mathrm{D}_{2} \mathrm{O}, 400 \mathrm{MHz}\right): \delta 5.85(\mathrm{~m}$, $\left.1 \mathrm{H}, \mathrm{CH}=\mathrm{CH}_{2}\right), 5.27-5.17\left(\mathrm{~m}, 2 \mathrm{H}, \mathrm{CH}=\mathrm{CH}_{2}\right), 5.19$, $5.03,5.02,4.94,4.93(5 \mathrm{~s}, 5 \mathrm{H}, 5 \mathrm{H}-1) ;{ }^{13} \mathrm{C}$ NMR $(100$ $\left.\mathrm{MHz}, \mathrm{D}_{2} \mathrm{O}\right): 133.52\left(1 \mathrm{C}, \mathrm{CH}_{2} \mathrm{CH}=\mathrm{CH}_{2}\right), 118.71$ $\left.\mathrm{C}, \mathrm{CH}_{2} \mathrm{CH}=\mathrm{CH}_{2}\right), 102.23,102.23,100.7,98.12,97.50$ (5 C-1). MALDI-TOF MS calcd for $\mathrm{C}_{33} \mathrm{H}_{56} \mathrm{O}_{26}$ : 868.79 [M]. found: $891.65(\mathrm{M}+\mathrm{Na})^{+}$.

\subsection{Allyl $\alpha$-D-mannopyranosyl-(1 $\rightarrow 2)-\alpha$-D-manno- pyranosyl-( $1 \rightarrow 6)$-[ $\alpha$-D-mannopyranosyl- $(1 \rightarrow 2)-\alpha$-D- mannopyranosyl-(1 $\rightarrow 2)]-\alpha$-D-mannopyranosyl-( $1 \rightarrow 6)$ - [ $\alpha$-D-mannopyranosyl-(1 $\rightarrow 2)]$ - $\alpha$-D-mannopyranosyl- $(1 \rightarrow 6)$-[ $\alpha$-D-mannopyranosyl-(1 $\rightarrow 2)-\alpha$-D-manno- pyranosyl- $(1 \rightarrow 2) \mid-\alpha$-D-mannopyranoside 2}

Compound 19 (386 mg, $0.08 \mathrm{mmol}$ ) was dissolved in an ammonia-saturated solution of 1:9 $\mathrm{CH}_{2} \mathrm{Cl}_{2}-$ $\mathrm{CH}_{3} \mathrm{OH}(120 \mathrm{~mL})$ at $40^{\circ} \mathrm{C}$. After $24 \mathrm{~h}$, the reaction mixture was concentrated to about $30 \mathrm{~mL}$, and then $\mathrm{CH}_{2} \mathrm{Cl}_{2}(100 \mathrm{~mL})$ was added. The resultant precipitate was filtered and washed four times with $\mathrm{CH}_{2} \mathrm{Cl}_{2}$ to afford 2 as white solid $(127 \mathrm{mg}, 95 \%):[\alpha]_{\mathrm{D}}+35.8$ (c 1.0, $\left.\mathrm{H}_{2} \mathrm{O}\right) ;{ }^{1} \mathrm{H}$ NMR $\left(\mathrm{D}_{2} \mathrm{O}, 400 \mathrm{MHz}\right): \delta 5.94(\mathrm{~m}$, $\left.1 \mathrm{H}, \mathrm{CH}=\mathrm{CH}_{2}\right), 5.35-5.25\left(2 \mathrm{H}, \mathrm{CH}=\mathrm{CH}_{2}\right), 5.25-$ $5.02(10 \mathrm{H}-1) ;{ }^{13} \mathrm{C}$ NMR $\left(100 \mathrm{MHz}, \mathrm{D}_{2} \mathrm{O}\right): 133.6(1$ $\left.\mathrm{C}, \quad \mathrm{CH}_{2} \mathrm{CH}=\mathrm{CH}_{2}\right), \quad 118.7 \quad\left(1 \quad \mathrm{C}, \quad \mathrm{CH}_{2} \mathrm{CH}=\mathrm{CH}_{2}\right)$, $101.97,101.97,101.97,101.97,100.46,100.38,98.11$, 97.96, 97.96, 97.39 (10 C-1). MALDI-TOF MS calcd for $\mathrm{C}_{63} \mathrm{H}_{106} \mathrm{O}_{51}: 1679.50[\mathrm{M}]$. found: $1702.61(\mathrm{M}+\mathrm{Na})^{+}$

\section{Acknowledgements}

This work was supported by the Beijing Natural Science Foundation (6021004). 


\section{References}

1. Goodman, D. S.; Teplitz, E. D.; Wishner, A.; Klein, R. S.; Burk, P. G.; Hershenbaum, E. J. Am. Acad. Dermatol. 1987, 17, 210-218.

2. Bodey, G. P. Am. J. Med. 1984, 77, 13-22.

3. Spebar, M. J.; Pruitt, B. A. J. Trauma 1981, 21, 237-243.

4. Silverman, S. J.; Luangjarmekorn, L.; Greenspan, D. J. Oral Med. 1984, 39, 194-201.

5. Nakajima, T.; Ballou, C. E. J. Biol. Chem. 1974, 249, 7679-7684.

6. Suzuki, S.; Shibata, N.; Kobayashi, H. In Fungal Cell Wall and Immune Response, NATA ASI Ser.; Latgé, J. P.; Boucias, D., Eds.; Springer-Verlag: Berlin, 1991; Vol. H53, pp. 111-121.

7. Kobayashi, H.; Kojimahara, T.; Takahashi, K.; Takihawa, M.; Takahashi, S.; Shibata, N.; Okawa, Y.; Suzuki, S. Carbohydr. Res. 1991, 214, 131-145.

8. Kanbe, T.; Cutler, J. E. Infect. Immun. 1994, 62, 1662 1668.
9. Stratford, M. Yeast 1992, 8, 635-645.

10. Nelson, R. D.; Shibata, N.; Podzorski, R. P.; Herron, M. J. Clin. Microbiol. Rev. 1991, 4, 1-19.

11. Podzorski, R. P.; Gray, G. R.; Nelson, R. D. J. Immunol. 1990, 144, 707-716.

12. Ning, J.; Yi, Y.; Kong, F. Tetrahedron Lett. 2002, 43, 5545-5549.

13. Ning, J.; Wang, H.; Yi, Y. Tetrahedron Lett. 2002, 43, 7349-7352.

14. Wang, H.; Ning, J. J. Org. Chem. 2003, 68, 2521-2524.

15. Ning, J.; Heng, L.; Kong, F. Tetrahedron Lett. 2002, 43, $673-675$.

16. Ning, J.; Heng, L.; Kong, F. Carbohydr. Res. 2002, 337, 1159-1164.

17. Kobayashi, H.; Komido, M.; Watanabe, M.; Matsuda, K.; Ikeda-Hasebe, T.; Suzuki, M.; Oyamada, H.; Shibata, N.; Suzuki, S. Infect. Immun. 1994, 62, 44254431.

18. Heng, L.; Ning, J.; Kong, F. J. Carbohydr. Chem. 2001, 20, 285-296. 